\title{
Free Convolution and the Random Sum of Matrices
}

By

\author{
Roland SPEICHER*
}

\begin{abstract}
We show that the spectral measure of the sum of two selfadjoint matrices is 'almost surely' given by the free convolution (in the sense of Voiculescu) of the spectral measures of the two matrices if their size tends to infinity.
\end{abstract}

\section{$\S 1$. Introduction}

Assume we know the spectral measures $\mu_{A}$ and $\mu_{B}$ of two $n \times n$-matrices $A$ and $B$. What can we say about the spectral measure of the sum of $A$ and $B$ ? One way to answer such a question is to average over all possibilities which are compatible with the given information.

If we knew that the two matrices commute, then we would average over all possibilities for choosing two diagonal matrices with the given spectral measures. The result would then be the convolution of the measures $\mu_{A}$ and $\mu_{B}$.

But here we want to consider the case where we have no additional information (like commutativity) about the relation between $A$ and $B$. Then the canonical procedure is to choose two fixed (e.g., diagonal) matrices $A$ and $B$ with the given spectral measures and average over all spectral measures of $A+U B U^{*}$ for all unitary matrices $U$, where the averaging measure for $U$ is the Haar measure on the group $U(n)$. The question is, how the resulting average measure is connected with the starting measures $\mu_{A}$ and $\mu_{B}$. For finite $n$ the connection seems to be very complicated, but in the limit where $n$ tends to $\infty$ it gets handable again. Indeed, it was shown by Voiculescu [Voi 3] that in this limit the averaging procedure gives a measure which is the free convolution of $\mu_{A}$ and $\mu_{B}$. This free convolution (and the corresponding notion of 'free independence') has a nice algebraic characterization and was introduced

Communicated by H. Araki, January 21, 1992.

1991 Mathematics Subject Classification : 46L50, 60G50, 60H25.

* Institut für Angewandte Mathematik, Universität Heidelberg. Im Neuenheimer Feld 294, D-69120 Heidelberg, Federal Republic of Germany.

This work has been supported by the Deutsche Forschungsgemeinschaft (SFB 123). 
by Voiculescu [Voi 1] in a totally different context. He also recognized that this new kind of convolution can be treated very analogously to the usual convolution and he developed an analytic aparatus for free convolution comparable to the usual Fourier transformation machinery for the usual convolution ([Voi 2], see also [Maa, Spe]). To sum it up, free convolution can be defined and handled easily without any reference to averaging procedures for matrices.

The aim of this paper is to improve Voiculescu's result in the respect, that the above statement is not only true for an average over all possible sums, but also for almost all possibilities itself. Thus we shall prove (informally spoken): If $A$ and $B$ are two selfadjoint infinite matrices with spectral measures $\mu_{A}$ and $\mu_{B}$, then the spectral measure of a typical sum $A+U B U^{*}$ is for almost all unitary $U$ given by the free convolution of $\mu_{A}$ and $\mu_{B}$. Of course, the notion of infinite matrices and 'almost all' has to be given a precise meaning by approximating $A, B$ and $U$ by finite-dimensional $n \times n$-matrices $A_{n}, B_{n}$ and $U_{n}$.

The problem of a direct, more appealing, formulation in terms of operators on an infinite-dimensional Hilbert space $\mathscr{H}$ suffers from the absence of a canonical measure on the group of all unitary operators on $\mathscr{H}$.

For technical reasons, we shall only deal with the case where $\mu_{A}$ and $\mu_{B}$ have compact support, because we can then restrict to the consideration of moments. However, we believe that the result is also true in the non-compact case, see [Maa] for an elegant treatment of free convolution in this case.

We hope that our result supports our philosophy that it is not too bad to model two (or more) operators, the relation among which is obscured to us because of initial lack of knowledge or because of a complex dynamics, by operators which are freely independent.

The paper is organized as follows. In the next chapter we shall recall the definition and some basic facts about free independence and free convolution and fix our notations for later use. The third chapter contains the formulation and the proof of our main theorem.

\section{$\S 2$. Some Facts about Free Independence and Fixing of Notations}

The following definitions of 'free independence' and 'free convolution' are essentially taken from [Voi3].

Let $\mathcal{C}$ be a unital $*$-algebra and $\varphi$ a state on $\mathcal{C}$, i. e. a positive linear functional with $\varphi(1)=1$. A family of subalgebras $\mathcal{C}_{\imath} \subset \mathcal{C}(i \in I)$ with $1 \in \mathcal{C}_{i}$ is called a free family of subalgebras (or $\mathcal{C}_{\imath}$ are called freely independent) in $(\mathcal{C}, \varphi)$ if $\varphi\left(a_{1} \cdots a_{n}\right)=0$ whenever we have $a_{1}, \cdots, a_{n}$ such that $a_{j} \leqq \mathcal{C}_{\imath(j)}$ with $i(j) \neq i(j+1)$ for all $1 \leqq j \leqq n-1$ and $\varphi\left(a_{k}\right)=0$ for all $1 \leqq k \leqq n$.

Given pairs $\left(\mathcal{C}_{k}, \varphi_{k}\right)(k \equiv J)$ of unital $*$-algebras $\mathcal{C}_{k}$ and states $\varphi_{k}$ on $\mathcal{C}_{k}$, 
there exists a state $\varphi$ on the free product algebra $\mathcal{C}:=\underset{k \in J}{\star C_{k}}$ (with identification of the units) such that $\varphi \mid \mathcal{C}_{k}=\varphi_{k}$ and such that the subalgebras $\left(\mathcal{C}_{k}\right)_{k \in J}$ form a free family of subalgebras in $(C, \varphi)$. The state $\varphi$ is uniquely determined by these conditions and is called the free product of the states $\varphi_{k}$.

Note that the above characterization of free independence allows to express all moments $\varphi\left(b_{1} \cdots b_{n}\right)$ whenever $b_{j} \in \mathcal{C}_{\imath(\jmath)}$ as polynomials in moments of the form $\varphi\left(b_{k_{1}} \cdots b_{k_{r}}\right)$ with $i\left(k_{1}\right)=i\left(k_{2}\right)=\cdots=i\left(k_{r}\right)$, i. e. $b_{k_{1}} \cdots b_{k_{r}} \in \mathcal{C}_{\imath\left(k_{1}\right)}$. In particular, we have stochastic independence between the different subalgebras in the sense $\varphi\left(a_{1} a_{2}\right)=\varphi\left(a_{1}\right) \varphi\left(a_{2}\right)$ whenever $a_{1} \in \mathcal{C}_{\imath(1)}, a_{2} \in \mathcal{C}_{\imath(2)}$ and $i(1) \neq i(2)$. But note also that the polynomial can be quite complicated for other moments, e. $g$. for $a \in \mathcal{C}_{1}, b \in \mathcal{C}_{2}$ we have

$$
\varphi(a b a b)=\varphi(a) \varphi(a) \varphi(b b)+\varphi(a a) \varphi(b) \varphi(b)-\varphi(a) \varphi(a) \varphi(b) \varphi(b) .
$$

By $C\left\langle X_{1}, \cdots, X_{s}\right\rangle$ we denote the free algebra with the $s$ generators $X_{1}, \cdots, X_{s}$, i. e. all polynomials in $s$ non-commuting indeterminants. Let now two probability measures $\mu_{1}$ and $\mu_{2}$ on $R$ (with compact support) be given. Then $\mu_{2}$ can be regarded as the distribution of the random variable $X_{2}=X_{\imath}^{*}$ on $\left(\boldsymbol{C}\left\langle X_{\imath}\right\rangle, \varphi_{\imath}\right)$, i. e. $\varphi_{\imath}$ is determined by

$$
\varphi_{i}\left(X_{i}^{k}\right)=\int x^{k} d \mu_{\imath}(x) \quad \text { for all } k \in \boldsymbol{N}_{0} .
$$

Now consider the free product $\varphi_{1} \star \varphi_{2}$ on $\boldsymbol{C}\left\langle X_{1}\right\rangle \star C\left\langle X_{2}\right\rangle=C\left\langle X_{1}, X_{2}\right\rangle$. The random variables $X_{1}$ and $X_{2}$ (i.e. the $*$-algebras generated by them) are freely independent in $\left(\boldsymbol{C}\left\langle X_{1}, X_{2}\right\rangle, \varphi_{1} \star \varphi_{2}\right)$ and we denote by $\mu_{1} \boxplus \mu_{2}$ the distribution of $X_{1}+X_{2}$ with respect to $\varphi_{1} \star \varphi_{2}$, i. e. $\mu_{1} \boxplus \mu_{2}$ is determined by

$$
\int x^{k} d\left(\mu_{1} \boxplus \mu_{2}\right)(x)=\varphi_{1} \star \varphi_{2}\left(\left(X_{1}+X_{2}\right)^{k}\right) \quad \text { for all } k \in \boldsymbol{N}_{0} .
$$

The probability measure $\mu_{1} \boxplus \mu_{2}$ is called the free convolution of $\mu_{1}$ and $\mu_{2}$. By the above remark, the moments of $\mu_{1} \boxplus \mu_{2}$ are polynomials in the moments of $\mu_{1}$ and $\mu_{2}$. It is clear that $\mu_{1} \boxplus \mu_{2}$ also has compact support. Free convolution can also be defined without problems when the support of $\mu_{1}$ or $\mu_{2}$ is not compact, cf. [Maa].

Free independence and free convolution behave in some respects quite analogous to the usual independence and convolution (cf. [Voil, 2, Spe]), but one should note that the free product is a completely non-commutative concept. An unusual feature of free convolution is the fact that $\boxplus$ is not distributive, i.e. in general $\mu \boxplus\left(\lambda \nu_{1}+(1-\lambda) \nu_{2}\right) \doteq \lambda \mu \boxplus \nu_{1}+(1-\lambda) \mu \boxplus \nu_{2}$.

In the rest of this chapter we shall fix our notations for the following.

We shall use the following probability spaces: Let $\Omega_{n}$ be the probability space of all $n \times n$-matrices, where all entries are independent, complex-valued, Gaussian distributed random variables with mean zero and variance $1 / n$. By 
$\Omega_{\text {gauss }}$ we denote the product space of all $\Omega_{n}$ (equipped with the canonical product measure), i. e.

$$
\Omega_{\text {gauss }}:=\bigotimes_{n=1}^{\infty} \Omega_{n} .
$$

In a similar way, we denote by $\Omega_{\text {unitary }}$ the product space of random sequences of unitary matrices

$$
\Omega_{\text {unitary }}:=\bigotimes_{n=1}^{\infty} \Omega_{n}^{\text {unitary }},
$$

where $\Omega_{n}^{\text {unitary }}$ is the group $U(n)$ of unitary $n \times n$-matrices equipped with the normalized Haar measure.

If $\mu_{\imath}(i \in \boldsymbol{N})$ and $\mu$ are probability measures on $\mathbb{R}$, then we denote by $\mu_{n} \stackrel{w}{\rightarrow} \mu$ the weak convergence of $\mu_{2}$ to $\mu$. We recall that in the case where $\mu$ has compact support it is determined by its moments and it is sufficient to check the convergence of all moments (cf. [Bil, Theorem 30.2]).

By $M_{n}$ we denote the complex *-algebra of $n \times n$-matrices, equipped with the normalized trace $\operatorname{tr}_{n}$ given by

$$
\operatorname{tr}_{n}(A):=\frac{1}{n} \sum_{i=1}^{n} a_{\imath \imath} \quad \text { for } A=\left(a_{j k}\right)_{j, k=1, \ldots, n} \in M_{n} .
$$

The adjoint of a matrix $A$ is denoted by $A^{*}$. By $|A|:=\left(A^{*} A\right)^{1 / 2}$ we denote the absolute value of $A \in M_{n}$. The expression $\prod_{l=1}^{n} A_{2}$ means the product $A_{1} \cdots A_{n}$ in the 'right' order.

For some selfadjoint $R \in M_{n}$ we denote by $\mu_{R}$ the spectral measure of $R$ with respect to the trace $\operatorname{tr}_{n}$, i. e. $\mu_{R}$ is determined by

$$
\operatorname{tr}_{n}\left(R^{k}\right)=\int x^{k} d \mu_{R}(x) \quad \text { for all } k \in \boldsymbol{N}_{0} .
$$

In connection with central limit theorems for the free independence it was seen [Voil, Spe] that the limiting states can be described as free Gaussian states on some $C\left\langle X_{1}, \cdots, X_{s}\right\rangle$ in the following way: Let $C V=\left\{V_{1}, \cdots, V_{p}\right\}$ be a partition of the set $\{1, \cdots, r\}$, i.e. the $V$, are ordered and disjoint sets and $\{1, \cdots, r\}$ $=\cup_{i=1}^{p} V_{\imath}$. Then $C V$ is called non crossing [Kre], if for all $i, j=1, \cdots, p$ with $V_{\imath}=\left(v_{1}, \cdots, v_{n}\right)\left(v_{1}<\cdots<v_{n}\right)$ and $V_{\jmath}=\left(u_{1}, \cdots, w_{m}\right)\left(w_{1}<\cdots<w_{m}\right)$ we have

$$
w_{k}<v_{1}<w_{k+1} \Longleftrightarrow w_{k}<v_{n}<w_{k+1} \quad(k=1, \cdots, m-1) .
$$

(In [Spe] we used the term 'admissible' instead of 'non crossing'.) We will denote the set of all partitions of $\{1, \cdots, r\}$ by $\mathscr{Q}(1, \cdots, r)$ and the set of all non crossing partitions by $\mathscr{P}_{N C}(1, \cdots, r)$. We can reformulate the definition of 'non crossing' in a recursive way: The partition $\mathcal{C}=\left\{V_{1}, \cdots, V_{p}\right\}$ is non crossing if at least one of the $V_{\imath}$ is a segment of $(1, \cdots, r)$, i.e. it has the form $V_{\imath}=(k, k+1, k+2, \cdots, k+m)$ and $\left\{V_{1}, \cdots, V_{\imath-1}, V_{\imath+1}, \cdots, V_{p}\right\}$ is a non 
crossing partition of $\{1, \cdots, r\} \backslash V$, (interpreted in a canonical way). We shall especially have to consider non crossing partitions $C V=\left\{V_{1}, \cdots, V_{p}\right\}$ where each $V_{\imath}$ contains exactly two elements. We shall write $V_{\imath}=\left(e_{\imath}, z_{\imath}\right)$ with $e_{\imath}<z_{\imath}$. The set of all such partitions will be denoted by $\mathscr{L}_{N C}^{2}(1, \cdots, r)$, where of course $r$ has to be of the form $r=2 m$.

A state $\varphi$ on $C\left\langle X_{1}, \cdots, X_{s}\right\rangle$ is called a free Gaussian state, if we have for all $r \in N$ and $k(1), \cdots, k(r) \in\{1, \cdots, s\}$

$$
\begin{aligned}
& \varphi\left(X_{k(1)} \cdots X_{k(r)}\right)
\end{aligned}
$$

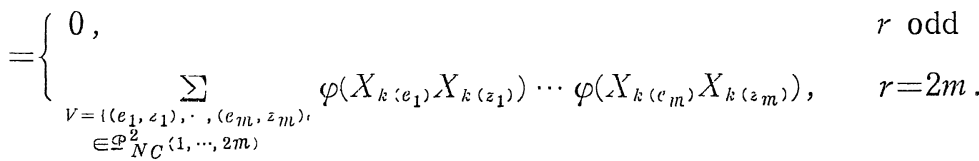

The matrix $\left(\varphi\left(X_{2} X_{\jmath}\right)_{2, j=1,, s}\right.$ is called the colariance matrix of $\varphi$.

\section{s. Statement and Proof of the Main Theorem}

Theorem. Let $\mathcal{A}=\left(\mathcal{A}_{n}\right)_{n \in N}$ and $\mathcal{B}=\left(B_{n}\right)_{n \in N}$ be two sequences of selfadjoint $n \times n$-matrices $1_{n}$ and $B_{n}$ such that

$$
\mu_{A_{n}} \stackrel{w}{\longrightarrow} \mu_{\llcorner l} \text { and } \quad \mu_{B_{n}} \stackrel{w}{\longrightarrow} \mu_{B}
$$

for some probability mesures $\mu_{\mathrm{L}}$ and $\mu_{\mathbb{S B}}$ on $\boldsymbol{l}_{\boldsymbol{L}}$. If $\mu_{\mathrm{L}}$ and $\mu_{\mathrm{SB}}$ have compact support, then

$$
\mu_{A_{n}+U_{n} B_{n} U_{n}} \stackrel{u^{\prime}}{\longrightarrow} \mu_{\mathcal{A}} \mathbb{A} \mu_{\mathcal{B}}
$$

for almost all random sequences of unitary matrices $U=\left(U_{n}\right)_{n \in N} \in \Omega_{\mathrm{unitar}}$.

The rough idea of the proof is the same as in [Voi3], we only have to adapt some arguments to the 'almost sure' situation.

The idea is the following: One first shows that infinitely large random matrices $\mathscr{X} \in \Omega_{\text {gauss }}$ and non-random diagonal matrices are freely independent, which implies also the free independence between polynomials in $\mathscr{X}$ and diagonal matrices. Then one notices that the phase $\mathcal{U}:=\mathscr{X}\left(\mathscr{X}^{2} * \mathscr{X}\right)^{-1 / 2}$ of a random matrix $\mathscr{X} \in \Omega_{\text {gauss }}$ gives an element $\mathcal{U} \in \Omega_{\text {unitary }}$ (under respectation of the measure) and tries to approximate $U$ by polynomials in $\mathcal{X}$. In this way one also gets the free independence between random unitary matrices $U \in \Omega_{\text {unitar }}$ and diagonal matrices. But free independence between $\{A, \mathscr{B}\}$ and $\left\{\mathscr{U}, \mathcal{U}^{*}\right\}$ implies free independence between $\mathcal{A}$ and $\mathscr{U} \mathscr{B} \mathcal{V}^{*}$.

The crucial observation made by Voiculescu is that not only $n \times n$-matrices with independent entries become freely independent in the limit $n \rightarrow \infty$, but that one can also include non-random diagonal matrices in this argument. This 
allows to start in our theorem with arbitrary spectral measures and not only with free Gaussian measures. To say it in another way, large matrices do not only give an interpretation of Wigner's semi-circle distribution, but much more generally for the concept of 'free independence'.

We shall now start with proving the free independence between random matrices in $\Omega_{\text {gauss }}$ and non-random diagonal matrices.

Lemma. For almost all $\mathfrak{X}=\left(X_{n}\right)_{n \in N} \in \Omega_{\text {gauss }}$ we have:

i) Let $P$ be a polynomial in lwo non-commuting indeterminants. Then

$$
\lim _{n \rightarrow \infty} \operatorname{tr}_{n}\left(P\left(X_{n}, X_{n}^{*}\right)\right)=\varphi\left(P\left(X, X^{*}\right)\right),
$$

where $\varphi$ is a free Gaussian state on $C\left\langle X, X^{*}\right\rangle$ with the covariance matrix $\left(\begin{array}{ll}0 & 1 \\ 1 & 0\end{array}\right)$.

ii) Let $\mathscr{D}^{1}=\left(D_{n}^{1}\right)_{n \in N}, \cdots, \mathscr{D}^{s}=\left(D_{n}^{s}\right)_{n \in N}$ be $s$ sequences of diagonal $n \times n$ matrices $D_{n}^{3}$ such that

$$
\lim _{n \rightarrow \infty} \operatorname{tr}_{n}\left(P\left(D_{n}^{1}, \cdots, D_{n}^{s}\right)\right)=\rho\left(P\left(D^{1}, \cdots, D^{s}\right)\right)
$$

for all polynomials $P$ in s indeterminants, where $\rho$ is a state on $C\left\langle D^{1}, \cdots, D^{s}\right\rangle$. Then

$$
\lim _{n \rightarrow \infty} \operatorname{tr}_{n}\left(P\left(X_{n}, D_{n}^{1}, \cdots, D_{n}^{s}, X_{n}^{*}\right)\right)=\rho \leftarrow \varphi\left(P\left(X, D^{1}, \cdots, D^{s}, X^{*}\right) j\right.
$$

for all polynomials $P$ in $(s+2)$ non-commuting indeterminants, where $\rho \star \varphi$ is the free product of $\rho$ and $\varphi$ on $C\left\langle X, D^{1}, \cdots, D^{s}, X^{*}\right\rangle$.

Remarks.

1) Clearly, i) is contained in ii). The main aim of the first part is to introduce the state $\varphi$.

2) One should note that $\varphi$ restricted to $\boldsymbol{C}\left\langle X^{*} X\right\rangle$ gives the 'square' of Wigner's semi-circle distribution (cf. [Voil, 3, Spe]), i.e.

$$
\lim _{n \rightarrow \infty} \operatorname{tr}_{n}\left(\left(X_{n}^{*} X_{n}\right)^{k}\right)=\frac{1}{\pi} \int_{-2}^{+2}\left(x^{2}\right)^{k} \sqrt{1-(x / 2)^{2}} d x .
$$

Proof of Lemma. It is sufficient to consider the monomials $X_{n}^{\imath(1)} \cdots X_{n}^{2(r)}$ for all choices of $r \in \boldsymbol{N}$ and $i(k) \in\{0,1, \cdots, s, s+1\}$, where

$$
\begin{aligned}
X_{n}^{0} & :=X_{n} \\
X_{n}^{j} & :=D_{n}^{j} \quad(1 \leqq j \leqq s) \\
X_{n}^{s+1} & :=X_{n}^{*} .
\end{aligned}
$$

Let in the following $r$ and $i(1), \cdots, i(r)$ be fixed and consider the random variable 


$$
\begin{aligned}
S_{n} & :=\operatorname{tr}_{n}\left(X_{n}^{\imath(1)} \cdots X_{n}^{\imath(1)}\right) \\
& =\frac{1}{n} k_{1}, \sum_{k_{r}=1}^{n} X_{n}^{\imath, 1}\left(k_{1}, k_{2}\right) X_{n}^{\imath(2)}\left(k_{2}, k_{3}\right) \cdots X_{n}^{\prime \prime}{ }^{\prime)}\left(k_{r}, k_{1}\right),
\end{aligned}
$$

where $X_{n}^{(j)}(k, l)$ is the $(k, l)$-entry in the matrix $X_{n}^{\prime}{ }^{j)}$.

Firstly, we shall show that the expectation value $\mathbb{E}\left[S_{n}\right]$ converges to the asserted expression. Secondly, we shall give an argument which guarantees also the almost sure convergence.

The first part is already treated in [Voi3], we shall only add to this treatment an 'explicit' formula for the limit. That $\rho \star \varphi$ takes on the same value in this case can also be seen easily, but we omit the proof of this since it follows from [Voi3].

We shall show that the limit for $S_{n}$ has the following form: Let us call a non crossing partition $C V=\left\{V_{1}, \cdots, V_{p}\right\}$ of $\{1, \cdots, r\}$ valid if one of the following two conditions is fulfilled:

(1) We have $p=1$ and $i(v) \in\{1, \cdots, s\}$ for all $v=1, \cdots, r$-in this case we deline $\rho \star \varphi(C V):=\rho\left(D^{\imath(1)} \cdots D^{\imath(r)}\right)$.

(2) The partition $Q v$ contains a segment $V_{\imath}$, i. e. $V_{\imath}=(v, v+1, \cdots, v+m)$, such that

$$
\begin{aligned}
i(v), i(v+m) & \in\{0, s+1\} \\
i(v) & \neq i(v+m) \\
i(v+1), \cdots, i(v+m-1) & \in\{1, \cdots, s\}
\end{aligned}
$$

and such that $C V \backslash\left\{V_{2}\right\}$ is a valid partition of $\{1, \cdots, r\} \backslash V_{1}$. In this second case we define

$$
\rho: \varphi\left(V_{1}\right):=\varphi\left(X^{\imath(v)} X^{\imath(v+m)}\right) \rho\left(D^{\imath(v+1)} \cdots D^{\imath(v+m-1)}\right)
$$

and recursively

$$
\rho * \varphi(C V):=\rho * \varphi\left(V_{1}\right) \cdot \rho * \varphi\left(C V \backslash\left\{V^{r}\right\}\right) .
$$

For example, for $C V=\{(1,9),(2,3,7,8),(4,5,6)\}$ (where $\left.\left.i_{1}^{\prime} 1\right), i(9) \notin\{0, s+1\}\right)$ we have

$$
\left.\rho \star \varphi^{\prime}(\mathcal{V})=\varphi\left(X^{\imath(2)} X^{\imath(8)}\right) \rho^{\left(D^{(3)}\right.} D^{\imath(7)}\right) \varphi\left(X^{\imath(1)} X^{\imath(6)}\right) \rho\left(D^{\imath(5)}\right) \rho\left(D^{\imath(1)} D^{\imath(9)}\right) .
$$

If we now denote the set of al! valid partitions of $\{1, \cdots, r\}$ by $\mathscr{P}_{v}(1, \cdots, r)$, then we shall show that almost surely

$$
\lim _{n \rightarrow \infty} S_{u}=\sum_{\neg \in \subseteq} \sum_{v(1, \cdots, r)} \rho \star \varphi(C V) .
$$

Now let us first consider the expectation value of $S_{n}$. For getting a contribution of the tuple $\left(k_{1}, \cdots, k_{r}\right)$ to

$$
\mathrm{E}\left[S_{n}\right]=\frac{1}{n} k_{1}, \sum_{, k_{7}=1}^{n} \mathrm{E}\left[X_{n}^{\prime \prime 1 \prime}\left(k_{1}, k_{2}\right) \cdots X_{n}^{\prime \prime r}\left(k_{r}, k_{1}\right)\right]
$$


the tuple must be such that each appearing matrix element of $X_{n}$ occurs at least twice in the product (under consideration of $X_{n}^{*}(k, l)=\bar{X}_{n}(l, k)$ ), because different entries of the matrices $X_{n}$ are independent and have expectation zero.

Denote by $I$ the set of the positions of the $X_{n}$ and $X_{n}^{*}$, i. e.

$$
I:=\{k \mid i(k) \in\{0, s+1\}\} .
$$

Given an index-tuple $\left(k_{1}, \cdots, k_{r}\right)$, we put $k_{r+1}:=k_{1}$ and call a pair $\left(k_{\jmath}, k_{\jmath+1}\right)$ for $1 \leqq j \leqq r$ a step of $\left(k_{1}, \cdots, k_{r}\right)$. We shall refine this notation by calling the pair

- a free step, if $i(j) \in\{0, s+1\}$ and $k_{\jmath+1}$ has not appeared before, i. e. $k_{\jmath+1}$ $\neq k_{l}$ for all $l$ with $1 \leqq l \leqq j$.

- a null step, if $i(j) \in\{1, \cdots, s\}$ and $k_{\jmath}=k_{j+1}$.

- a repetive step, if $i(j) \in\{0, s+1\}$ and $k_{\jmath+1}$ has appeared before, i. e. there is a $l$ with $1 \leqq l \leqq j$ such that $k_{j+1}=k_{l}$.

We say that two steps $\left(k_{\jmath}, k_{\jmath+1}\right)$ and $\left(k_{l}, k_{l+1}\right)$ agree, if $i(j), i(l) \in\{0, s+1\}$ and

$$
\begin{cases}\left(k_{\jmath}, k_{\jmath+1}\right)=\left(k_{l}, k_{l+1}\right), & \text { if } i(j)=i(l) \\ \left(k_{\jmath}, k_{\jmath+1}\right)=\left(k_{l+1}, k_{l}\right), & \text { if } i(j)=z i(l) .\end{cases}
$$

Now one sees as in [Wig] that we only have to consider index-tuples $\left(k_{1}, \cdots, k_{r}\right)$ which have the following properties: They contain only free, null or repetive steps and the number of the free steps is equal to the number of the repetive steps, namely they have no choice but to be $|I| / 2$. This implies that in such a tuple each repetive step $\left(k_{\jmath}, k_{j+1}\right)$ has to agree with exactly one prior free step $\left(k_{l}, k_{l+1}\right)$. Let us call such a tuple a valid one. Combinatorial arguments show that the contribution of all non valid tuples vanishes in the limit, their sum is at most of order $1 / n$ (cf. [Wig]).

Now consider a valid tuple $\left(k_{1}, \cdots, k_{r}\right)$. It defines a valid partition $C V$ in the following way: Let $m$ be the smallest number such that $\left(k_{m}, k_{m+1}\right)$ is a repetive step. Then this step has to be equal to exactly one prior free step, say $\left(k_{l}, k_{l+1}\right)$ with $l<m$. This is only possible if $\left(k_{l+1}, k_{l}\right)=\left(k_{m}, k_{m+1}\right)$ (i. e. a $X_{n}$ is paired with a $X_{n}^{*}$ and vice versa) and if all steps between $l$ and $m$ are null steps. Then we define $V_{1}:=(l+1, \cdots, m)$ and take this part from $k_{l+1}$ to $k_{m}$ out of our tuple $\left(k_{1}, \cdots, k_{r}\right)$. Since $k_{l}=k_{m+1}$, we get again a valid tuple and we can repeat the above procedure. But in this way we exactly build up in a recursive way a valid partition and it is easy to see that the summation over all tuples resulting in the same valid partition $Q V$ gives the contribution $\rho \star \varphi(C V)$ to $\lim _{n \rightarrow \infty} \mathrm{E}\left[S_{n}\right]$. Since on the other hand all valid partitions can appear, the assertion is proved.

It remains to prove that $S_{n}$ converges also almost surely. This can be done by showing a sufficient good estimate for the variance $\operatorname{Var}\left[S_{n}\right]$ of the form 


\section{(*) $\quad \operatorname{Var}\left[S_{n}\right] \leqq \frac{c}{n^{2}}$,}

where the constant $c$ is independent of $n$. This is sufficient because one can conclude

$$
\mathrm{E}\left[\sum_{n=1}^{\infty}\left(S_{n}-\mathrm{E}\left[S_{n}\right]\right)^{2}\right]=\sum_{n=1}^{\infty} \operatorname{Var}\left[S_{n}\right]<\infty
$$

which implies

$$
\sum_{n=1}^{\infty}\left(S_{n}-\mathrm{E}\left[S_{n}\right]\right)^{2}<\infty \quad \text { almost surely }
$$

and therefore

$$
\lim _{n \rightarrow \infty}\left(S_{n}-\mathrm{E}\left[S_{n}\right]\right)=0 \quad \text { almost surely. }
$$

Thus it remains to prove (*). We have

$$
\begin{aligned}
& \operatorname{Var}\left[S_{n}\right]=\mathrm{E}\left[S_{n}^{2}\right]-\mathrm{E}\left[S_{n}\right]^{2} \\
& =\frac{1}{n^{2}} k_{k_{1}}, \sum_{k_{1}=1}^{\infty} \sum_{l_{1}}^{\infty}, l_{l}=1 \\
& \left\{\mathrm{E}\left[X_{n}^{l^{(1)}}\left(k_{1}, k_{2}\right) \cdots X_{n}^{(1)}\left(k_{r}, k_{1}\right) X_{n}^{\imath^{(1)}}\left(l_{1}, l_{2}\right) \cdots Y_{n}^{\prime(r)}\left(l_{r}, l_{1}\right)\right]-\right. \\
& \left.\mathrm{E}\left[X_{n}^{2(1)}\left(k_{1}, k_{2}\right) \cdots Y_{n}^{2(r)}\left(k_{r}, k_{1}\right)\right] \cdot \mathrm{E}_{[}\left[X_{n}^{\imath^{(1)}}\left(l_{1}, l_{2}\right) \cdots X_{n}^{\left({ }^{(1)}\right.}\left(l_{r}, l_{1}\right)\right]\right\} .
\end{aligned}
$$

For all tuples $\left(k_{1}, \cdots, k_{r}\right),\left(l_{1}, \cdots, l_{r}\right)$ where each step of $\left(k_{1}, \cdots, k_{r}\right)$ does not agree with any step of $\left(l_{1}, \cdots, l_{r}\right)$ the $k$-factors are independent of the $l$-factors, hence such tuples make no contribution. Now consider on the contrary those tuples where at least one step of $\left(k_{1}, \cdots, k_{r}\right)$ agrees with one step of $\left(l_{1}, \cdots, l_{r}\right)$. There are finitely many possibilities which $k$-step agrees with which $l$-step. We fix one of these possibilities, say we consider the set of all tuples where $\left(k_{m}, k_{m+1}\right)$ agrees with $\left(l_{\jmath}, l_{\jmath+1}\right)$ for fixed $m$ and $j$ with $1 \leqq m, j \leqq r$. Of course, we can restrict to the case that both of these pairs belong to a $X_{n}$ or a $X_{n}^{*}$, i. e. that $i(m), i(i) \in\{0, s+1\}$.

Consider furthermore first only such tuples with $i, m)=i(i)$, hence $\left(k_{m}, k_{m+1}\right)$ $=\left(l_{\jmath}, l_{\jmath+1}\right)$. Then we can rewrite

$$
X_{n}^{(1)}\left(k_{1}, k_{2}\right) \cdots X_{n}^{u(r)}\left(k_{r}, k_{1}\right) X_{n}^{u(1)}\left(l_{1}, l_{2}\right) \cdots Y_{n}^{u(r)}\left(l_{r}, l_{1}\right)
$$

as

$$
\begin{aligned}
& Y_{n}^{\imath \prime m}\left(k_{m}, k_{m+1}\right) \cdots X_{n}^{\imath(r)}\left(k_{r}, k_{1}\right) X_{n}^{2(1)}\left(k_{1}, k_{2}\right) \cdots X_{n}^{\imath(m-1)}\left(k_{m-1}, k_{m}\right) \\
& Y_{n}^{\langle\jmath\rangle}\left(l_{\jmath}, l_{\jmath+1}\right) \cdots X_{n}^{\langle(r)}\left(l_{r}, l_{1}\right) X_{n}^{(1)}\left(l_{1}, l_{2}\right) \cdots X_{n}^{(\langle\jmath-1)}\left(l_{\jmath-1}, l_{\jmath}\right)
\end{aligned}
$$

and apply our results from the first part of this proof to the $2 r$-tuple

$$
\left(k_{m}, \cdots, k_{r}, k_{1}, \cdots, k_{m-1}, k_{m}=l_{1}, l_{\jmath+1}, \cdots, l_{r}, l_{1}, \cdots, l_{\jmath-1}\right) .
$$

They say that all our considered tuples are not valid (since $i(m)=i(j)$ means a 
pairing of a $X_{n}$ with a $X_{n}$ or a pairing of a $X_{n}^{*}$ with a $X_{n}^{*}$ ), which results in a sum of order $1 / n^{2}$ (note that the factor $1 / n$ in our considerations on $\mathrm{E}\left[S_{n}\right]$ is now replaced by $\left.1 / n^{2}\right)$.

Now consider the case $i(m) \neq i(j)$, which means $\left(k_{m}, k_{m+1}\right)=\left(l_{j+1}, l_{\jmath}\right)$. In this case our problem is equivalent to the consideration of the $2 r$-tuple

$$
\left(k_{m+1}, \cdots, k_{r}, k_{1}, \cdots, k_{m}, k_{m+1}=l_{j}, l_{\jmath+1}, \cdots, l_{r}, l_{1}, \cdots, l_{\jmath-1}\right) .
$$

But again such tuples are not valid, because the steps $\left(k_{n}, k_{m+1}\right)$ and $\left(l_{\jmath}, l_{\jmath+1}\right)$ agree, but both are repetive steps $\left(k_{m+1}\right.$ has appeared before as first element of the sequence and $l_{\jmath+1}$ has appeared before as $k_{m}$ ), whereas in valid tuples always repetive steps are paired with free steps. Thus again the contribution of this case is of order $1 / n^{2}$.

All in all, the sum of all tuples for which the $k$-factors and the $l$-factors are not independent can be estimated as less than $c / n^{2}$, where $c$ is some combinatorial factor independent of $n$. This finishes the proof.

Now we can prove our theorem.

Proof of Theorem. First, we can assume without restriction of generality that all $A_{n}$ and $B_{n}$ are diagonal matrices: We can diagonalize $A_{n}$ and $B_{n}$ in the form $A_{n}=V_{n} A_{n}^{d} V_{n}^{*}$ and $B_{n}=W_{n} B_{n}^{d} W_{n}^{*}$. Then

$$
\mu_{A_{n}+U_{n} B_{n} U_{n}^{\star}}=\mu_{A_{n}^{d}+\left(V_{n}^{*} U_{n} W_{n}^{*}\right) B_{n}^{d}\left(V_{n}^{\star} U_{n} W_{n}\right)^{*}}
$$

and the assertion is equivalent to the assertion for $\mathcal{A}^{d}=\left(A_{n}^{d}\right)_{n \in N}$ and $\mathscr{B}^{d}=$ $\left(B_{n}^{d}\right)_{n \in N}$, since the map $U_{n} \mapsto V_{n}^{*} U_{n} W_{n}$ preserves the Haar measure on $U(n)$.

Let

$$
\begin{aligned}
& F: \Omega_{\text {gauss }} \longrightarrow \Omega_{\text {unitary }} \\
& \mathscr{X}=\left(X_{n}\right) \longmapsto \mathscr{U}=\left(U_{n}\right)
\end{aligned}
$$

be the measurable mapping defined by

$$
U_{n}:=F\left(X_{n}\right):=X_{n}\left(X_{n}^{*} X_{n}\right)^{-1 / 2},
$$

which is defined almost everywhere. Since the measure on $\Omega_{\text {unitary }}$ is the image measure under this mapping of the measure on $\Omega_{\text {gauss }}$ (cf. [Voi3]), it is sufficient to prove

$$
\mu_{A_{n}+F\left(X_{n}\right) B_{n} F^{(}\left(X_{n}\right) *} \stackrel{w}{\longrightarrow} \mu_{\iota} \boxplus \mu_{\mathscr{B}}
$$

for almost all $\mathscr{X}=\left(X_{n}\right)_{n \in N} \in \Omega_{\text {gauss }}$.

Choose now such an $\mathscr{X}=\left(X_{n}\right)_{n \in N} \in \Omega_{\text {gauss }}$ where we can assume that $U_{n}:=$ $X_{n}\left(X_{n}^{*} X_{n}\right)^{-1 / 2}$ is defined for all $n \in \boldsymbol{N}$. Since $\mu_{\mathfrak{l}}$ and $\mu_{\mathscr{B}}$, and hence $\mu_{\mathfrak{A}} \boxplus \mu_{\mathscr{B}}$, have compact support, they are determined by their moments and it suffices to show the convergence of all moments of $\mu_{A_{n}+U_{n} B_{n} U_{n}^{3}}$ to the corresponding mo- 
ments of $\mu_{\mathcal{A}} \boxplus \mu_{\mathscr{B}}$. This is guaranteed if we check the characterizing property of free independence, namely: Let $P_{1}, \cdots, P_{2 r}$ be polynomials in one indeterminant such that for all $i=1, \cdots, r$

$$
\lim _{n \rightarrow \infty} \operatorname{tr}_{n}\left(P_{2 \iota-1}\left(A_{n}\right)\right)=0
$$

and

$$
\lim _{n \rightarrow \infty} \operatorname{tr}_{n}\left(P_{2 \imath}\left(U_{n} B_{n} U_{n}^{*}\right)\right)=\lim _{n \rightarrow \infty} \operatorname{tr}_{n}\left(P_{2 \imath}\left(B_{n}\right)\right)=0 .
$$

Then we have to show

$$
\begin{aligned}
\lim _{n \rightarrow \infty} \operatorname{tr}_{n}\left(\prod_{i=1}^{r} P_{2 \imath-1}\left(A_{n}\right) P_{2 \imath}\left(U_{n} B_{n} U_{n}^{*}\right)\right) & \\
= & \lim _{n \rightarrow \infty} \operatorname{tr}_{n}\left(\prod_{\imath=1}^{r} P_{2 \iota-1}\left(A_{n}\right) U_{n} P_{2 \iota}\left(B_{n}\right) U_{n}^{*}\right)=0 .
\end{aligned}
$$

We shall show this by approximating $U_{n}$ by polynomials in $X_{n}$ and $X_{n}^{*}$. One knows that $\lim _{n \rightarrow \infty}\left\|X_{n}\right\|=2$ almost surely icf. e.g. [Sil, Gem]), i. e. we can assume that there exists an $M \in \boldsymbol{R}$, such that $\left\|X_{n}\right\| \leqq M$ for all $n \in \boldsymbol{N}$. Choose now for $\varepsilon>0$ a polynomial $g_{\varepsilon}$ with real coefficients which is a good approximation of $1 / \sqrt{x}$ in the sense that we have

$$
\frac{1}{\pi} \int_{-2}^{+2}\left(1-g_{\varepsilon}\left(x^{2}\right) x\right)^{2} \sqrt{1-(x / 2)^{2}} d x \leqq \varepsilon \quad \text { for some fixed } l \text { with } 4 r \leqq 2^{l}
$$

and

$$
\sup _{x \in\left[-M^{2}, M^{2}\right]}\left|x g_{\varepsilon}(x)^{2}\right| \leqq 1
$$

Now put

$$
Q_{\varepsilon}\left(X_{n}, X_{n}^{*}\right):=X_{n} g_{\varepsilon}\left(X_{n}^{*} X_{n}\right)
$$

Then we can write

$$
U_{n}=Q_{s}\left(X_{n}, X_{n}^{*}\right)+R_{n}^{(\varepsilon)},
$$

where $Q_{\varepsilon}\left(X_{n}, X_{n}^{*}\right)$ is a polynomial in $X_{n}$ and $X_{n}^{*}$, whose norm can be estimated as

$$
\left\|Q_{\varepsilon}\left(X_{n}, X_{n}^{*}\right)\right\|^{2}=\left\|g_{\varepsilon}\left(X_{n}^{*} X_{n}\right) X_{n}^{*} X_{n} g_{\varepsilon}\left(X_{n}^{*} X_{n}\right)\right\| \leqq 1,
$$

and $R_{n}^{(s)}$ is an $n \times n$-matrix, which is small in the following sense:

$$
\begin{aligned}
\operatorname{tr}_{n}\left(\left|R_{n}^{(s)}\right|^{2 l}\right) & =\operatorname{tr}_{n}\left(\left(R_{n}^{(\varepsilon) *} R_{n}^{(\varepsilon)}\right)^{2^{l-1}}\right) \\
& =\operatorname{tr}_{n}\left(\left\{\left[\left(X_{n}^{*} X_{n}\right)^{-1 / 2}-g_{\varepsilon}\left(X_{n}^{*} X_{n}\right)\right] X_{n}^{*} X_{n}\left[\left(X_{n}^{*} X_{n}\right)^{-1 / 2}-g_{\varepsilon}\left(X_{n}^{*} X_{n}\right)\right]\right\}^{2^{l-1}}\right) \\
& =\operatorname{tr}_{n}\left(\left\{\left[1-g_{\varepsilon}\left(X_{n}^{*} X_{n}\right)\left(X_{n}^{*} X_{n}\right)^{1 / 2}\right]\left[1-\left(X_{n}^{*} X_{n}\right)^{1 / 2} g_{\varepsilon}\left(X_{n}^{*} X_{n}\right)\right]\right\}^{2^{l-1}}\right) .
\end{aligned}
$$

By the second remark after our lemma and by the fact that $\varphi$ on $C\left\langle X^{*} X\right\rangle$ is determined by its moments we have for all continuous, bounded $f$ on $\boldsymbol{R}$ 


$$
\lim _{n \rightarrow \infty} \operatorname{tr}_{n}\left(f\left(X_{n}^{*} X_{n}\right)\right)=\frac{1}{\pi} \int_{-2}^{+2} f\left(x^{2}\right) \sqrt{1-(x / 2)^{2}} d x .
$$

Since we have $\left\|X_{n}^{*} X_{n}\right\| \leqq M^{2}$ for all $n \in N$, we can even take any continuous function $f$ on $\boldsymbol{R}$ and thus we get

$$
\lim _{n \rightarrow \infty} \operatorname{tr}_{n}\left(\left|R_{n}^{\prime s)}\right|^{2 l}\right)=\frac{1}{\pi} \int_{-2}^{+2}\left(1-g_{\varepsilon}\left(x^{2}\right) x\right)^{2 l} \sqrt{1-(x / 2)^{2}} d x \leqq \varepsilon .
$$

Since in all monomials in the polynomial $Q_{s}\left(X_{n}, X_{n}^{*}\right)$ the number of $X_{n}$ and the number of $X_{n}^{*}$ is different, we can infer from our lemma that

$$
\lim _{n \rightarrow \infty} \operatorname{tr}_{n}\left(Q_{\varepsilon}\left(X_{n}, X_{n}^{*}\right)\right)=0 .
$$

Now we have

$$
\begin{aligned}
\lim _{n \rightarrow \infty} \operatorname{tr}_{n}\left(\prod_{i=1}^{r} P_{2 \ell-1}\left(A_{n}\right) U_{n} P_{2 \imath}\left(B_{n}\right) U_{n}^{*}\right) & \\
= & \lim _{n \rightarrow \infty} \operatorname{tr}_{n}\left(\prod_{\imath=1}^{r} P_{2 \imath-1}\left(A_{n}\right) Q_{\varepsilon}\left(X_{n}, X_{n}^{*}\right) P_{2 \imath}\left(B_{n}\right) Q_{\varepsilon}\left(X_{n}, X_{n}^{*}\right)^{*}\right) \\
& \quad+\lim _{n \rightarrow \infty} \sum \operatorname{tr}_{n}\left(\prod_{i=1}^{r} P_{2 \imath-1}\left(A_{n}\right) S_{n}^{(2 \imath-1)} P_{2 \imath}\left(B_{n}\right) S_{n}^{(2 \imath)}\right),
\end{aligned}
$$

where in the first term all $U_{n}$ are replaced by $Q_{\varepsilon}\left(X_{n}, X_{n}^{*}\right)$ and the sum in the second term runs over all $S_{n}{ }^{11}, \cdots, S_{n}^{(2 r)} \in\left\{Q_{\varepsilon}\left(X_{n}, X_{n}^{*}\right), R_{n}^{(s)}\right\}$, where at least one $S_{n}^{(j)}$ is equal to $R_{n}^{(\varepsilon)}$.

By our lemma, the first term vanishes in the limit $n \rightarrow \infty$. (One should note that one needs indeed a little stronger version of the lemma, because we can not be sure that the joint distribution of $A_{n}$ and $B_{n}$ exists in the limit $n \rightarrow \infty$. But since our $Q_{s}\left(X_{n}, X_{n}^{*}\right)$ has the special property that it really seperates $A_{n}$ from $B_{n}$ in the sense that in each monomial of $Q_{\varepsilon}\left(X_{n}, X_{n}^{*}\right)$ the number of $X_{n}$ is different from the number of $X_{n}^{*}$, the valid partitions in the calculation of the considered term never combine a $\downarrow_{n}$ with a $B_{n}$, i.e. the joint distribution is not really needed. For the argument that the non-valid terms do not contribute it is sufficient to know that the possibly appearing joint moments of $A_{n}$ and $B_{n}$ remain bounded as $n$ goes to $\infty$. But this is guaranteed by the assumption of a compact support for $\mu_{\llcorner}$and $\mu_{\mathscr{B}}$.)

A summand in the second term can be estimated as follows: From

$$
\left|\operatorname{tr}_{n}(A B)\right|^{2} \leqq \operatorname{tr}_{n}\left(|A|^{2}\right) \operatorname{tr}_{n}\left(|B|^{2}\right) \quad\left(.4, B \in M_{n}\right)
$$

we can deduce by induction

$$
\left|\operatorname{tr}_{n}\left(A_{1} \cdots A_{k}\right)\right|^{2 l} \leqq \operatorname{tr}_{n}\left(\left|A_{1}\right|^{2 l}\right) \cdots \operatorname{tr}_{n}\left(\left|A_{k}\right|^{2}\right)
$$

for all $k \leqq 2^{l}$ and $A_{1}, \cdots, A_{k} \in M_{n}$. This implies for our fixed $l$ with $4 r \leqq 2^{l}$ 


$$
\begin{aligned}
& \left.\mid \operatorname{tr}_{n}\left(\prod_{\imath=1}^{r} P_{2 \imath-\mathrm{i}}\left(A_{n}\right) S_{n l}^{2 \ell-1} P_{2 \imath}\left(B_{n}\right) S_{n}^{(2 \imath)}\right)\right)\left.\right|^{2 l} \\
& \quad \leqq \prod_{\imath=1}^{r} \operatorname{tr}_{n}\left(\left|P_{2 \ell-1}\left(4_{n}\right)\right|^{2^{\ell}}\right) \operatorname{tr}_{n}\left(\left|P_{2 \iota}\left(B_{n}\right)\right|^{2^{l}}\right) \cdot \prod_{\jmath=1}^{2 r} \operatorname{tr}_{n}\left(\left|S_{n}^{(\jmath)}\right|^{2^{l}}\right)
\end{aligned}
$$

(Note that $\operatorname{tr}_{n}\left(\left|S_{n}^{(j),}\right|^{2 l}\right)=\operatorname{tr}_{n}\left(\left|S_{n}^{(j)}\right|^{2 l}\right)$.) In the limit we can estimate these factors as follows:

$$
\begin{aligned}
& \lim _{n \rightarrow \infty} \prod_{i=1}^{r} \operatorname{tr}_{n}\left(\left|P_{2 \imath-1}\left(A_{n}\right)\right|^{2^{l}}\right)=\prod_{\imath=1}^{r} \int\left|P_{2 \imath-1}(x)\right|^{\left.\right|^{l}} d \mu_{\llcorner}(x)=: M_{\llcorner}<\infty \\
& \lim _{n \rightarrow \infty} \prod_{i=1}^{r} \operatorname{tr}_{n}\left(\left|P_{2 \imath}\left(B_{n}\right)\right|^{2^{l}}\right)=\prod_{\imath=1}^{r} \int\left|P_{2 \imath}(x)\right|^{2^{l}} d \mu_{\mathscr{B}}(x)=: M_{\mathscr{B}}<\infty .
\end{aligned}
$$

If $S_{n}^{(\jmath)}=Q_{\varepsilon}\left(X_{n}, X_{n}^{*}\right.$; then we have

$$
\operatorname{tr}_{n}\left(\left|S_{n}^{\left.()^{\prime}\right)}\right|^{2^{l}}\right) \leqq\left\|Q_{\varepsilon}\left(X_{n}, X_{n}^{*}\right)\right\|^{2 l} \leqq 1,
$$

and for $S_{n}^{(\jmath)}=R_{n}^{(\varepsilon)}$ we get

$$
\lim _{n \rightarrow \infty} \operatorname{tr}_{n}\left(\left|S_{n l}^{\jmath^{\prime}}\right|^{2^{l}}\right)=\lim _{n \rightarrow \infty} \operatorname{tr}_{n}\left(\left|R_{n}^{\varepsilon^{\prime}}\right|^{2^{l}}\right) \leqq \varepsilon .
$$

Since at least one of the $S_{n}^{\prime \prime}$ is equal to $R_{n}^{(\varepsilon)}$ we get for $\varepsilon \leqq 1$

$$
\left|\lim _{n \rightarrow \infty} \operatorname{tr}_{n}\left(\prod_{\imath=1}^{r} P_{2 \ell-1}\left(.1_{n}\right) U_{n} P_{2 \imath}\left(B_{n}\right) U_{n}^{*}\right)\right| \leqq\left(2^{2 r}-1\right) \sqrt[2^{l}]{\varepsilon M_{\llcorner} \downarrow l M_{\mathcal{B}}} .
$$

Since this is valid for all $\varepsilon$ with $0<\varepsilon \leqq 1$ and since $M_{A}$ and $M_{\mathscr{B}}$ are independent of $\varepsilon$ we arrive finally at

$$
\lim _{n \rightarrow \infty} \operatorname{tr}_{n}\left(\prod_{i=1}^{r} P_{2 \ell-1}\left(A_{n}\right) U_{n} P_{2 \imath}\left(B_{n}\right) U_{n}^{*}\right)=0,
$$

which we wanted to show.

\section{References}

[Bil $]$ Billingsley, P., Probability and Measure, John Wiley \& Sons, New York, 1979.

[Gem] Geman, S., A limit theorem for the norm of random matrices, Ann. Probab., 8 (1980), 252-261.

[Kre J Kreweras, G., Sur les partitions non croisees d'un cycle, Discr. Math., 1 (1972), 333-350.

-Maa ] Maassen, H., Addition of Freely Independent Random Variables, J. Funct. Anal., 106 (1992), 409-438.

rSil I Silverstein, J.W.. Eigenvalues and eigenvectors of large dimensional sample covariance matrices, Contemporary Mathematics, 50 (1986), 153-160.

-Spe ] Speicher, R., A New Example of 'Independence' and 'White Noise', Probab. Th. Rel. Fields, 84 (1990), 141-159.

-Voi1] Voiculescu, D., Symmetries of some reduced free product $C^{*}$-algebras, in $O$ perator 
Algebras and their Connection with Topology and Ergodic Theory, Lecture Notes in Mathematics 1132, Springer Verlag, Heidelberg 1985, 556-588.

[Voi2] Voiculescu, D., Addition of certain non-commuting random variables, J. Funct. Anal., 66 (1986), 323-346.

[Voi3] — Limit laws for random matrices and free products, Invent. math., 104 (1991), 201-220.

[Wig] Wigner, E.P., Characteristic vectors of bordered matrices with infinite dimensions, Ann. of Math., 62 (1955), 548-564. 